\title{
RECHERCHES SUR LA FAUNE HELMINTHOLOGIQUE \\ DE L'INDOCHINE (CESTODES ET TRÉMATODES)
}

\author{
Par Ch. JOYEUX et E. HOUDEMER \\ (Suile el fin)
}

\section{PSEUDOPHYLLIDAE}

\section{Diphyllobothriidæ}

Scyphocephalus bisulcatus (Riggenbach, 1899). - Intestin de varan (Varanus sp.), région de Hanoï. Collections du laboratoire de Parasitologie.

Bothridium pithonis de Blainville, 1824. - Intestin de Python reticulatus Gray, Hanoī.

Bothridium pithonis var. minor Joyeux et Baer, 1926. - Intestin de Python reticulatus Gray. Paris, Muséum d'Histoire naturelle.

L'un de nous, en collaboration avec J.-G. Baer (1927), a récemment étudié les Bothridium de serpents africains et asiatiques, Nous avons montré qu'ils appartiennent à deux espèces distinctes et que les premières descriptions se rapportent à B. ovatum (Dies., 1850) pour les parasites africains, à $B$. pithonis Bl., 1824, pour les asiatiques. Nous avons créé une variété minor, à côté de l'espèce type, Bothridium pithonis.

Sparganum mansoni (Cobbold, 1883). - Historique. Sparganum mansoni (Cobbold, 1883) a été découvert en 1881 par Patrick Manson, à l'autopsie d'un Chinois, et décrit ensuite par Cobbold. Depuis cette époque, un grand nombre de plérocercoides ont été signalés, tant chez l'homme que chez les animaux et déterminés généralement comme "Sparganum mansoni ". Cette forme larvaire a été signalée surtout en Extrême-Orient; on y a aussi rattaché des parasites découverts en Afrique, en Australie, au Texas, en Guyane anglaise.

La diagnose exacte d'un Sparganum est, en l'état actuel de nos

(1) Nous rangeous la famille des Proteocephalidw dans les Tetraphyllidea suivant les idées généralement admises ; cependant Southwell (1925) a profondément modifié la classification de ce groupe, en se basant uniquement sur les caractères du scolex; les Proteocephalide passent des Tetraphyllidea dans les Cyclophyllidea.

Annales de Parasitologie, T. VI, $\mathrm{N}^{\circ} 1,-1^{\text {er }}$ janvier 1928, p. 27-58. 
connaissances, très difficile à poser, toutes ces larves ne possédant guère de carctères morphologiques permettant de les différencier. Le seul moyen d'arriver à une détermination juste est d'obtenir le ver adulte correspondant, par ingestion du Sparganum. Mais cette opération doit être suivie de l'identification précise du bothriocéphale développé expérimentalement; malheureusement les diverses espèces de Diphyllobothrium sont loin d'être toutes entièrement décrites. Il en résulte inévitablement une certaine confusion dans la morphologie et la biologie de ce groupe ; pour ce qui est de Sparganum mansoni, son histoire complète ne parait pas élucidée jusqu'à présent.

Railliet, Henry et Bauche ont signalé à Hué (Annam), dans les muscles du pore, un Sparganum qu'ils ont rattaché à $S$. mansoni, puis à $S$. raillietti Ratz, 1912, existant en Serbie et en Hongrie. L'adulte de ce dernier est bien connu, nous renvoyons au récent travail de Kotlan (1923), qui confirme les recherches de V. Ratz. Dans son mémoire récent (1924), Railliet pense qu'il pourrait s'agir du plérocercoïde de $D$. latum, hébergé, comme on sait, par divers poissons; le porc représenterait un hôte surnuméraire.

Plusieurs auteurs japonais ont obtenu expérimentalement le ver adulte en partant de Sparganum mansoni. Yamata (1916), avec un plérocercoïde trouvé dans la cavité abdominale d'une malade, a fait développer un ver dans l'intestin du chien; les œufs du bothriocéphale sont apparus dans les selles au $13^{\circ}$ jour. Yoshida (1917) fait ingérer au chat des Sparganum provenant d'autres chats; il observe également des œufs dans les selles au bout de 13 jours. Par l'examen de ces oufs, Yoshida distingue le bothriocéphale développé expérimentalement de $D$. latum, mais les caractères extérieurs et l'anatomie lui paraissent analogues chez les déux vers. Okumura (1919) élucide complètement le cycle évolutif de $S$. mansoni. Il montre l'ubiquité du plérocercoïde pouvant se trouver chez des batraciens (Rana nigromaculata), des serpents (Elaphe climacophora), expérimentalement chez des souris. Le procercoïde existe chez Cyclops leuckarti Sars. L'adulte peut se voir chez le chien, le chat, et d'autres carnivores. Le cycle évolutif de ce bothriocéphale est donc calqué sur celui de $D$. latum. Okumura observe, de plus, le phénomène du réencapsulement du plérocercoïde ; précédemment Hirohata et Maejima (in Yoshida, 1917) avaient déjà transplanté des scolex dans le péritoine du lapin. Muto et Miraha (1921) obtiennent aussi un bothriocéphale en partant d'une plérocercoïde de grenouille ; Yoshida (1922) prouve que les Sparganum de grenouille donnent un adulte identique à celui produit par les Sparganum de l'homme. R.-T. Leiper confirme ces 
expériences (1923) en faisant évoluer ün Sparganum de mangouste chez le chat, il obtient un bothriocéphale. Ando (1925) fait également développer chez le chien et le chat des Sparganum provenant d'homme, de chat, de poule, de grenouille. D'après cet auteur, le plérocercoïde peut être hébergé par le singe, le porc, le lapin, le cobaye, le rat, la souris, la poule, le pigeon et la grenouille. Dans certains villages du Japon, 95 p. 100 des chats et 20 p. 100 des chiens sont parasités par le ver adulte. Nous n'avons malheureusement pu lire les travaux de Muto et Miraha, de Yoshida, d'Ando, parus dans des périodiques japonais ; nous en avons seulement eu connaissance par les analyses du Tropical diseases Bulletin.

A Hué, Evanno (1927), en six expériences, infeste des chiens en partant 'de Sparganum d'homme, de grenouille, de couleuvre, et obtient chaque fois des bothriocéphales adultes. De plus, il inocule avec succès un Sparganum de grenouille dans le tissu conjonctif sous-cutané abdominal d'un singe. Enfin, il montre la possibilité de l'infestation par voie conjonctivale, déjà soupçonnée par Collin : déposant dans le cul-de-sac conjonctival d'un singe un petit Sparganum de 7 millimètres, provenant d'une grenouille, il pratique ensuite une blépharorraphie avec agrafes qui sont enlevées au bout de trois jours. A ce moment, le Sparganum est disparu. Onze jours après, il s'est formé une tumeur sous la peau de la paupière inférieure gauche. Par incision de celle-ci, on retrouve le Sparganum vivant qui a grandi et mesure 3 centimètres en contraction. Il est intéressant de noter que les empiriques indigènes prescrivent, pour diverses affections oculaires, l'application de grenouilles sur l'œil. Dans deux observations de Collin, il semble bien que cette pratique ait eu pour résultat de provoquer une sparganose, par le mécanisme ci-dessus démontré.

Reste à savoir à quelles espèces correspondent les adultes obtenus expérimentalement. Il s'agit vraisemblablement de Diphyllobothrium et, suivant les lois de la nomenclature, on les nomme Diphyllobothrium mansoni (Cobbold, 1883), dénomination qui a désigné, en réalité, la forme larvaire. A notre connaissance, aucune étude anatomique de $D$. mansoni n'a été faite jusqu'à présent, seuls les œufs ont été décrits et différenciés de ceux de $D$. latum par Yoshida. Récemment Ando (1925), Kobayashi (1925) ont émis l'hypothèse que $D$. mansoni était semblable à $D$. decipiens.

Recherches PERsonnelles. - Les Sparganum sont fréquents en Indochine. Comme dans tout l'Extrême-Orient, on les trouve chez de nombreux vertébrés : mammifères, oiseaux, reptiles, batraciens; nous avons peu de renseignements pour ce qui concerne les poissons. La sparganose humaine est bien connue, notamment 
la localisation oculaire : citons les travaux de Casaux (1914), de Motais (1920), de Collin (1925), d'Evanno (1927), etc. ; nous renvoyons à ces auteurs pour l'étude clinique de la maladie. Le nombre de cas humains traités en Annam augmente, d'après les chiffres de Collin, d'année en année : 9 cas en 1924, 16 en 1925, 28 en 1926. Il y aurait lieu de voir si ces chiffres correspondent à un réel accroissement de la sparganose, ou s'ils ne sont pas simplement en rapport avec l'augmentation générale des consultations données aux indigènes.

Voici la liste des animaux chez lesquels nous avons eu l'occasion de récolter des Sparganum; nous indiquons pour quelques-uns le pourcentage d'infestation.

Mammifères. Homme. Singes (16 0/0) : Macacus cynomolgus L., M. nemestrinus L. Rats : Epimys norvegicus (Klein), E. rattus L. $(3,50 / 0)$. Civette : Viverra zibetha $\mathrm{L}$.

Oiseaux. Poule domestique d'Indochine $\left(\begin{array}{lll}6,96 & 0 / 0\end{array}\right)$. Pigeon domestique d'Indochine $(2,50 / 0)$. Pintade : Numida meteagris L. $(750 / 0)$. Dryonastes perspicillata $\mathrm{Gm}$. (50 $0 / 0)$. Urocissa sinensis Gray. Upupa epops indica Hadgson. Gennæus nycthemerus L.

Il est à remarquer que nous n'avons jamais trouvé d'oiseaux aquatiques hébergeant des Sparganum. Evanno (1927) confirme également ces résultats négatifs.

Reptiles. Zamenis mucosus L. Naja tripudians Merrem. Bungarus fasciatus Schneider.

Batraciens. Rana tigrina Daud. $(800 / 0)$. Evanno le signale chez d'autres batraciens voisins.

D'après cet auteur, les poissons de rizières ne sont jamais parasités.

Nous nous sommes efforcés d'identifier ces parasites par l'expérimentation. Afin de voir s'ils correspondaient à une seule ou à plusieurs espèces adultes, nous sommes partis de Sparganum hébergés par des vertébrés zoologiquement éloignés les uns des autres ; soit mammifères (homme), oiseaux (poule, Dryonastes perspicillata), reptiles (Zamenis mucosus): Nous les avons fait ingérer par de jeunes chiens, isolés au moment du sevrage, et soigneusement tenus à l'abri de toute contamination.

Le tableau suivant résume nos expériences. Les Sparganum d'origine humaine, à localisation oculaire, ont été extraits par le $\mathrm{D}^{\mathrm{r}}$ Casaux, qui a déjà eu-l'occasion, en collaboration avec l'un de nous (Casaux et Houdemer, 1926), de publier les résultats préliminaires obtenus.

Nous estimons, après examen des adultes obtenus, qu'ils appar- 


\begin{tabular}{|c|c|c|c|c|}
\hline \multirow[b]{2}{*}{$\begin{array}{c}\text { Hôte } \\
\text { DU SPARGANUM }\end{array}$} & \multicolumn{3}{|c|}{ DATES } & \multirow[b]{2}{*}{ Obsernitions } \\
\hline & $\begin{array}{l}\text { de l'ingestion } \\
\text { par le chien }\end{array}$ & $\begin{array}{c}\text { de l'apparition } \\
\text { des cufs } \\
\text { dans les selles } \\
\text { du chien }\end{array}$ & $\begin{array}{l}\text { de l'autopsie } \\
\text { du chien }\end{array}$ & \\
\hline $\begin{array}{l}\text { Homme (spar- } \\
\text { ganose ocu- } \\
\text { laire)......... }\end{array}$ & 11 juin 1926. & $\begin{array}{r}24 \text { juin } 1926 \\
\text { (13 jours). }\end{array}$ & $\begin{array}{l}\text { 1er juillet } \\
1926 \quad(19 \\
\text { jours). }\end{array}$ & $\begin{array}{l}3 \text { Sparganum in } \\
\text { gérés. } \\
3 \text { Bothriocépha- } \\
\text { les obtenus. }\end{array}$ \\
\hline $\begin{array}{r}\text { Homme (spar- } \\
\text { ganose ocu- } \\
\text { laire)........ }\end{array}$ & 19 juin 1926. & $\begin{array}{c}28 \text { juin } 1926 \\
(9 \text { jours }) .\end{array}$ & $\begin{array}{l}22 \text { juillet } \\
1926 \text { (32 } \\
\text { jours). }\end{array}$ & $\begin{array}{l}3 \text { Sparganum in- } \\
\text { gérés dont } \\
\text { abîmé, } \\
2 \text { Bothriocépha- } \\
\text { les obtenus. }\end{array}$ \\
\hline Poule .......... & $\begin{array}{l}\text { 24 décembre } \\
1924 .\end{array}$ & - & $\begin{array}{l}30 \text { décembre } \\
1924 \quad(6 \\
\text { jours). }\end{array}$ & $\begin{array}{l}2 \text { Sparganum in- } \\
\text { gérés. } \\
1 \text { Bothriocéphale } \\
\text { obtenu. } \\
\text { Chien tué acci- } \\
\text { dentellement. }\end{array}$ \\
\hline $\begin{array}{r}\text { Dryonasles pers- } \\
\text { picillala...... }\end{array}$ & 31 mai 1925. & - & $\begin{array}{r}19 \text { juin } 1925 . \\
\text { (19 jours). }\end{array}$ & $\begin{array}{l}2 \text { Sparganum in- } \\
\text { gérés. } \\
1 \text { Bothriocéphale } \\
\text { obtenu. }\end{array}$ \\
\hline $\begin{array}{c}\text { Zamenis muco- } \\
\text { sus.......... }\end{array}$ & Septembre 1926. & - & - & $\begin{array}{l}4 \text { Sparganum in- } \\
\text { gèrés par } 2 \\
\text { chien. } \\
4 \text { Bothriocépha- } \\
\text { les obtenus. }\end{array}$ \\
\hline
\end{tabular}

tiennent tous à la même espèce. Dans le cas du bothriocéphale provenant d'un Sparganum de poule, nous n'avons eu qu'un très jeune ver, âgé de 6 jours, mesurant 9 millimètres de long sur 0,4 de largeur maxima, ne montrant aucune trace d'ébauche génitale. Le dianostic exact est donc impossible, cependant son scolex est absolument semblable à ceux décrits ci-dessous; nous pensons qu'il doit être identique à eux. 
DESCRIPTION DU BOTHRIOCÉPHALE ADULTE. - La longueur est variable dans d'assez larges limites. Elle oscille dans la plupart des échantillons autour de 60 centimètres, les derniers anneaux paraissant appartenir à un adulte bien formé. Dans un cas, nous avons noté 1 mètre, chez un animal en extension moyenne. La largeur maxima atteint 5 à 6 millimètres.

Dans presque toute l'étendue de la chaîne, les anneaux sont plus larges que longs ; à la fin, la longueur augmente et peut arriver à égaler la largeur, l'anneau étant alors carré. Voici la moyenne obtenue par plusieurs mensurations.

\begin{tabular}{|l|c|c||}
\hline & Longuger & Lírgetr \\
\cline { 2 - 3 } Partie médiane de la chaine ...... & 1 mill. & 5 mill. \\
Partie terminale de la chaîne...... & 5,25 & 5,5 \\
\hline
\end{tabular}

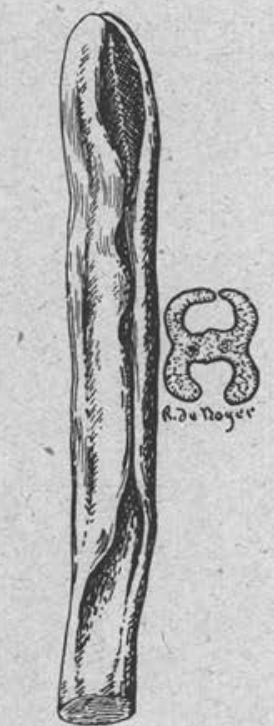

Fig. 7. - Scolex de $D i$ phyllobothrium mansoni. Vue d'ensemble et coupe transversale, avec les cordons nerveux.

La partie postérieure de chaque anneau recouvre légèrement le suivant, formant un bord libre en forme de volant, qu'on peut facilement soulever en introduisant dessous la pointe d'un scalpel. Ce bord libre, plus ou moins allongé, suivant la contraction, mesure 200 à $350 \mu$ de long.

Le scolex (fig. 7) a la forme allongée caractéristique des Diphyllobothrium; sa longueur est de 1 mill. à 1 mill., 5 , prise depuis l'extrémité supérieure du ver jusqu'à la terminaison des bothridies; sa largeur maxima varie de 0 mill., 4 à 0 mill., 8 , suivant l'état de contraction. Cette tête se distingue facilement de celle de D. latum. Elle est moins aplatie, presque quadrangulaire. Les cavités des bothridies-sont plus larges et les bords flottants plus marqués. Ils peuvent se replier l'un par-dessus l'autre dans les préparations; on obtient alors l'aspect figuré par Chandler pour $D$. decipiens (1925), où la fente paraît décrire une spire. Comme chez $D$. latum, les cavités correspondent aux faces dorsale et ventrale des anneaux, ainsi que le montre, en coupes transversales, la position des vaisseaux et surtout des nerfs, facilement visibles. Sur chacun des bords latéraux du scolex existe une petite dépression. 
Le cou est d'une importance extrêmement variable suivant l'état de contraction des échantillons. Sur l'un d'eux, en bonne extension, il mesure 16 mill., 5, depuis l'extrémité postérieure des bothridies jusqu'aux premières traces de segmentation. Sur d'autres, il n'existe pas et la segmentation se poursuit jusqu'au niveau de la tête.

La cuticule est très épaisse. La couche souscuticulaire est formée de cellules allongées, serrées les unes contre les autres. La musculature longitudinale est représentée par une seule couche de faisceaux très régulièrement disposés côte à côte, formant par endroits une bande ininterrompue ; ceux qui sont nettement individualisés possèdent une trentaine de fibres. La musculature transversale se compose de 5 ou 6 fibres fines, irrégulièrement concentriques. La musculature dorso-ventrale est peu développée.

Le système aquifère s'observe bien sur les anneaux jeunes, à organes génitaux imparfaitement développés. Il se présente sous l'aspect de huit troncs longitudinaux, quatre de chaque côté de la masse génitale centrale. Leur diamètre, en coupe transversale (fig. 8), atteint en moyenne 25 à $27 \mu$. A mesure qu'on avance dans la chaine des proglottis, on constate l'existence de vaisseaux secondaires de plus en plus considérables, émettant des ramifications transverses et se divisant d'une façon complexe. Il s'ensuit que le système aquifère devient de plus en plus difficile à interpréter à mesure qu'on l'examine dans des anneaux plus âgés. Dans les derniers anneaux, il y a environ 12 vaisseaux, tant principaux que secondaires, sans compter les petites ramifications; ce chiffre est d'ailleurs variable. Ils sont absents de la zone corticale.

Le système nerveux est représenté par deux gros troncs, passant de chaque côté de la masse génitale centrale, dont ils sont séparés par deux vaisseaux principaux, tandis que les deux autres sont situés de l'autre côté, vers le bord

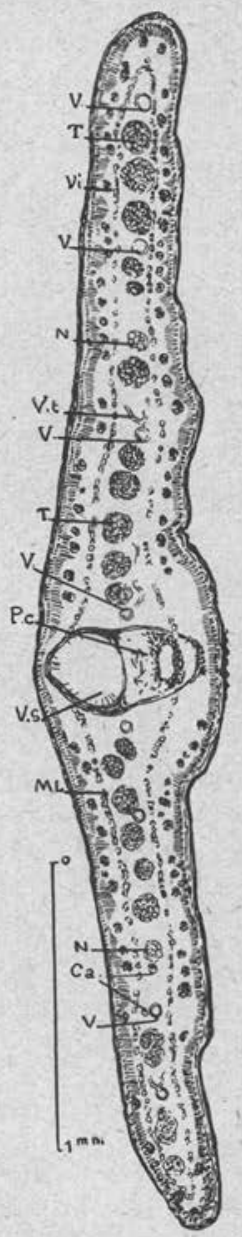

Fig. 8. - Coupe transversale d'un anneau de Diphyllobothrium mansoni. $\mathrm{V}$, vaisseaux longitudinaux ; Vt, vaisseanx transversaux ; N, nerf; T, testicules: Vi, vitellogène : Vs, vésicule séminale externe; Pc, poche du cirre; Ml, musculature longitudinale ; Ca, corpuscules calcaires. 
latéral. Dans chaque moitié d'anneau, le trone nerveux a done deux vaisseaux à sa droite et deux à sa gauche. Il est facilement visible, son diamètre est de 45 à $50 \mu$ sur 24 à $26 \mu$.

Les corpuscules calcaires sont assez nombreux dans le parenchyme cortical et médullaire ; ils mesurent en moyenne $10 \mu$ de diamètre.

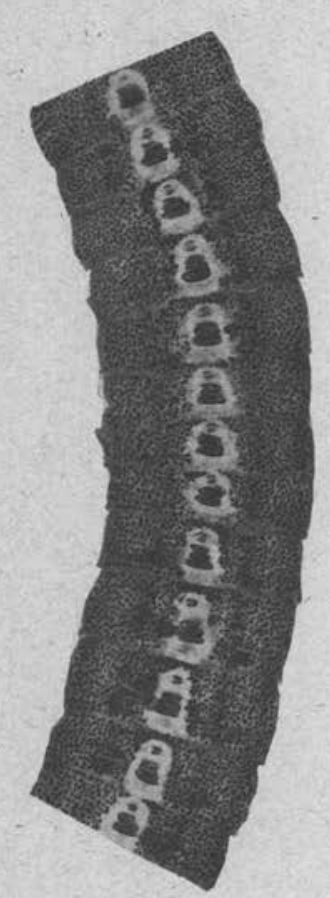

Fig. 9. - Microphotographie d'une série d'anneaux successifs de Diphyllobothrium mansoni montrant la disposition variable des vitellogènes.

Appareil mále. - Les testicules offrent une disposition très particulière. Le Diphyllobothrium que nous décrivons représente une forme de passage entre ceux chez lesquels les testicules sont rangés sur deux champs latéraux, ne se joignant pas à la partie antérieure de l'anneau et ceux chez lesquels existe la disposition inverse : rencontre des testicules en avant de l'orifice génital. Chez nos vers, on observe ces deux dispositions avec toutes les transitions (fig. 9).

Les vitellogènes offrent également cette irrégularité suivant les anneaux considérés.

Voici une statistique, faite, d'une part, sur de jeunes anneaux; d'autre part, sur des anneaux mûrs, indiquant les dispositions successives prises par les testicules et les vitellogènes. Nous les indiquons par les abréviations suivantes :

A, testicules et vitellogènes se rencontrant nettement sur plusieurs rangées à la partie antérieure de l'anneau.

$B$, testicules et vitellogènes se touchant à peine.

$\mathrm{C}$, testicules et vitellogènes sur deux champs latéraux, ne se touchant pas à la partie antérieure de l'anneau.

Chaîne de 74 anneaux successifs, jeunes.

$\mathrm{C}-\mathrm{C}-\mathrm{C}-\mathrm{C}-\mathrm{C}-\mathrm{C}-\mathrm{C}-\mathrm{C}-\mathrm{C}-\mathrm{C}-\mathrm{C}-\mathrm{C}-\mathrm{C}-$ $\mathrm{B}-\mathrm{A}-\mathrm{B}-\mathrm{A}-\mathrm{B}-\mathrm{A}-\mathrm{A}-\mathrm{B}-\mathrm{C}-\mathrm{B}-\mathrm{C}-\mathrm{B}-\mathrm{B}-$ $\mathrm{B}-\mathrm{C}-\mathrm{C}-\mathrm{C}-\mathrm{A}-\mathrm{C}-\mathrm{C}-\mathrm{C}-\mathrm{C}-\mathrm{B}-\mathrm{A}-\mathrm{B}-\mathrm{C}-$ $\mathrm{C}-\mathrm{C}-\mathrm{A}-\mathrm{A}-\mathrm{B}-\mathrm{C}-\mathrm{C}-\mathrm{C}-\mathrm{C}-\mathrm{C}-\mathrm{C}-\mathrm{C}-\mathrm{C}-$ $\mathrm{B}-\mathrm{A}-\mathrm{A}-\mathrm{A}-\mathrm{C}-\mathrm{B}-\mathrm{C}-\mathrm{C}-\mathrm{C}-\mathrm{B}-\mathrm{C}-\mathrm{C}-\mathrm{A}-$ $\mathrm{B}-\mathrm{C}-\mathrm{B}-\mathrm{C}-\mathrm{B}-\mathrm{C}-\mathrm{C}-\mathrm{C}-\mathrm{B}$. 
Chaîne de 83 anneaux successifs, portion terminale.

$\mathrm{B}-\mathrm{B}-\mathrm{A}-\mathrm{A}-\mathrm{A}-\mathrm{B}-\mathrm{A}-\mathrm{A}-\mathrm{A}-\mathrm{C}-\mathrm{C}-\mathrm{B}-$ $\mathrm{A}-\mathrm{A}-\mathrm{C}-\mathrm{A}-\mathrm{B}-\mathrm{C}-\mathrm{C}-\mathrm{C}-\mathrm{B}-\mathrm{C}-\mathrm{C}-\mathrm{C}-\mathrm{B}-$ $\mathrm{B}-\mathrm{C}-\mathrm{C}-\mathrm{C}-\mathrm{C}-\mathrm{B}-\mathrm{C}-\mathrm{A}-\mathrm{A}-\mathrm{A}-\mathrm{A}-\mathrm{A}-\mathrm{B}-$ $\mathrm{C}-\mathrm{B}-\mathrm{B}-\mathrm{C}-\mathrm{B}-\mathrm{B}-\mathrm{C}-\mathrm{C}-\mathrm{B}-\mathrm{C}-\mathrm{B}-\mathrm{B}-\mathrm{C}-$ $\mathrm{B}-\mathrm{B}-\mathrm{B}-\mathrm{B}-\mathrm{B}-\mathrm{B}-\mathrm{B}-\mathrm{C}-\mathrm{C}-\mathrm{C}-\mathrm{C}-\mathrm{C}-\mathrm{C}-$ $\mathrm{C}-\mathrm{C}-\mathrm{C}-\mathrm{C}-\mathrm{C}-\mathrm{C}-\mathrm{B}-\mathrm{C}-\mathrm{C}-\mathrm{C}-\mathrm{A}-\mathrm{C}-\mathrm{A}-$ $\mathrm{C}-\mathrm{C}-\mathrm{C}-\mathrm{B}-\mathrm{B}-\mathrm{C}$.

Soit, en additionnant les résultats :

\begin{tabular}{|c|c|c|}
\hline & ANNEAUX JEUNES & ANNEAUX Agés \\
\hline $\begin{array}{l}\text { Testicules et vitellogènes se tou } \\
\text { chant netlement }(\mathrm{A}) \ldots \ldots \ldots \ldots \ldots\end{array}$ & 12 & 16 \\
\hline $\begin{array}{l}\text { Testicules et vitellogènes se tou- } \\
\text { chant à peine (B) } \ldots \ldots \ldots \ldots \ldots \ldots\end{array}$ & 18 & 27 \\
\hline $\begin{array}{c}\text { Testicules et vitellogènes ne se } \\
\text { touchant pas (C).............. }\end{array}$ & 44 & 40 \\
\hline Totaux. & 74 & 83 \\
\hline
\end{tabular}

On voit que la réunion à la face antérieure de l'anneau a tendance à se manifester plutòt dans la portion terminale de la chaîne.

Dans ces conditions, le nombre de testicules est impossible à fixer, même d'une façon approximative. En employant la méthode généralement usitée, c'est-à-dire en les comptant sur une coupe transversale, puis sur une coupe sagittale, en multipliant ensuitè les deux chiffres l'un par l'autre, et en répétant cette opération à plusieurs endroits de la chaîne, nous obtenons :

Nombre de testicules, comptés sur des coupes transversales intéressant la masse génitale centrale : 20 à 30 , soit 10 à 15 de chaque côté de la masse génitale.

Nombre de testicules, comptés sur des coupes sagittales n'intéressant pas la masse génitale centrale : 16 à 18 .

Produits des multiplications : 320 à 540 testicules par anneau.

En coupe transversale, les testicules apparaissent sur une seule rangée ; cependant, quelquefois, sur les bords de l'anneau, ils chevauchent plus ou moins et l'on peut en observer deux, l'un derrière l'autre, dans le sens dorso-ventral. Ils sont arrondis ou ovalaires ; le diamètre de ceux qui sont régulièrement arrondis varie de 135 à $160 \mu$. 
Le canal déférent (fig. 10) décrit des sinuosités dans la partie de l'anneau antérieure au pore utérin ; il vient déboucher ensuite

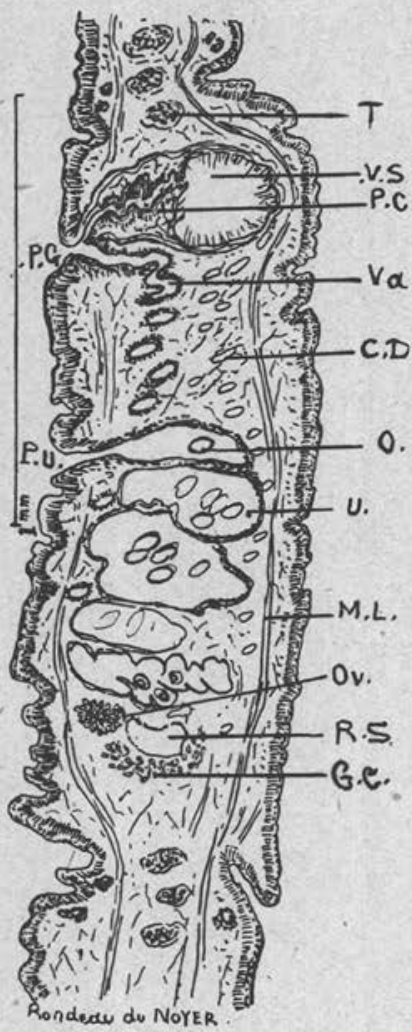

Fig. 10. - Coupe sagittale de Diphyllobothrium mansoni, cirre invaginé et vésicule séminale externe distendue. T, testicules; Vs, vésicule séminale externe; PC, poche du cirre; Va, vagin : CD, canal déférent ; O, œuf; U, utérus ; Ov, ovaire; RS, réceptacle séminal ; GC, glande coquillière ; ML, musculature longitudinale; $\mathrm{PG}$, pore génital ; PU, pore utérin. dans la vésicule séminale externe.

Les rapports entre la vésicule séminale externe et la poche du cirre varient avec l'état de contraction de cet appareil et suivant que le cirre est invaginé ou évaginé. Ces dispositions sont absolument comparables à celles qui ont été figurées par Nybelin pour diverses espèces de pseudophyllidés (1922).

La vésicule séminale est puissamment musclée; à l'intérieur elle est tapissée d'une couche de cellules ciliées, analogues à celles que Kotlan a décrites chez $D$. raillieti (1923). Sa lumière se continue directement avec celle du cirre. Elle occupe toute la zone médullaire de l'anneau, dans le sens dorso-ventral et atteint la bande musculaire longitudinale du côté dorsal. Lorsqu'elle est distendue (fig. 4), sa forme est globuleuse, ayant à peu près $280 \mu$ de diamètre. La poche du cirre se voit surtout lorsque le cirre est rétracté à son intérieur. On peut la comparer à un cône, dont le sommet serait formé par l'orifice génital mâle, et la base accolée à la vésicule séminale ; la hauteur, représentant la longueur de la poche, atteint environ $250 \mu$. Lorsque la vésicule séminale est contractée et le cirre évaginé (fig. 11), l'aspect est tout différent. Il est alors difficile d'identifier la poche du cirre qui se confond avec la vésicule séminale.

Le cirre est formé de tissu réticulaire, avec quelques fibres musculaires. L'orifice génital mâle est antérieur à l'orifice femelle. Ils sont très rapprochés l'un de l'autre et apparaissent contigus sur des coupes sagittales. Ils sont situés au $3 / 8$ de l'anneau, en comptant à partir du bord antérieur. L'atrium génital est peu marqué. 
A ppareil femelle. - La vulve se présente à la surface de l'anneau, sous forme d'une fente ovalaire ou fusiforme, dont le grand axe est transversal. Elle a à peu près $100 \mu$ de long.

Le vagin a un calibre plus ou moins large, suivant son état de contraction. En coupe sagittale, on le voit se diriger d'abord dans le sens ventro-dorsal ; puis, vers la moitié de l'anneau, il se courbe à angle droit et gagne la partiè postérieure du segment, en décrivant des sinuosités à droite et à gauche. Sur les coupes, les sections de ce conduit sont faciles à reconnaitre, elles ont une paroi plus épaisse que celle du canal déférent. Il contourne le pore utérin et

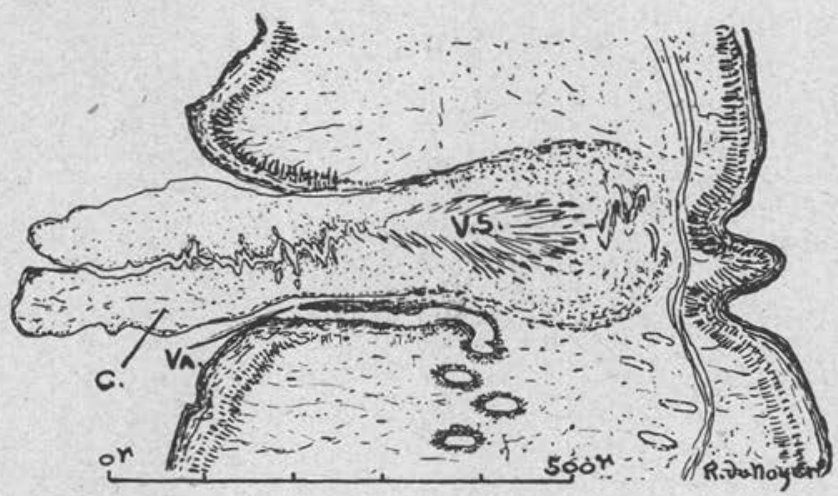

Fig. 11. - Portion d'une coupe sagittale de Diphyllobothrium mansoni montrant le cirre évaginé et la vésicule séminale externe contractée. C, cirre; VS, vésicule séminale externe; Va, vagin.

passe ventralement par rapport aux boucles de l'utérus. Arrivé à la partie postérieure de l'anneau, il se dilate en un réceptacle séminal, arrondi ou ellipsoïdique, ayant environ $85 \mu$ de diamètre. L'ovaire est situé en arrière de l'utérus, dans la partie postérieure de l'anneau. Il est bilobé, s'étendant à droite et à gauche de la ligne médiane. Sa largeur est de 1 mill., 4 pour un anneau de la portion terminale de la chaîne, mesurant lui-même 5 millimètres de largeur. La glande coquillière est centrale ; elle a une forme en croissant, à concavité dirigée vers la partie antérieure de l'anneau. Les cellules qui la composent apparaissent plus ou moins éosinophiles suivant les échantillons ; ces différences sont sans doute en rapport avec l'état de fonctionnement de la glande. Nous avons parlé de la disposition variable des vitellogènes, à propos des testicules; ils se trouvent dans le parenchyme cortical et s'étendent dans la portion de l'anneau qui recouvre le suivant. Dans la région postérieure débouchent les vitelloductes. 
L'utérus part de la portion postérieure du segment. Dans un anneau fixé en bonne extension, on le voit décrire trois boucles qui vont en décroissant d'ampleur à mesure qu'il se rapproche de l'orifice. La première boucle a environ $800 \mu$ de large, la troisième $650 \mu$. Naturellement, sur les anneaux contractés, cet aspect est plus difficile à reconnaître. On n'observe pas, autour de l'orifice utérin, de circonvolutions en forme de rosette, comme chez D. latum. L'extrémité de l'organe n'apparait pas bourrée d'œufs comme le reste du tube. La paroi est tapissée de cellules se colorant par l'hémalun, que nous interprétons comme les cellules glandulaires décrites par Nybelin (1922), quoiqu'elles n'aient pas l'aspect globuleux et piriforme, mais soient plutôt allongées. Elles ne sont pas toujours très marquées et peuvent disparaitre au niveau de la boucle supérieure. Nybelin indique d'ailleurs qu'elles sont très peu développées chez les Diphyllobothriidæ.

L'orifice utérin se trouve sur la ligne médiane, parfois légèrement dévié. Il a la forme d'une fente mesurant environ $240 \mu$ dans son grand axe, qui est transversal. Il est situé à une distance du pore génital variant suivant l'état de contraction de l'anneau ; nous avons noté 50 à $120 \mu$. En tous cas, l'orifice femelle est toujours plus rapproché de l'orifice mâle que de l'orifice utérin.

Les œufs sont operculés. Leurs dimensions ne sont pas constantes ; en mesurant ceux qui proviennent du même utérus, on trouve des variations assez considérables. Nous les avons examinés en dilacérant l'utérus d'un des derniers anneaux chez nos divers échantillons. Les chiffres extrêmes obtenus varient de 52 à $62 \mu$ sur 32 à $40 \mu$. En ne tenant compte que des œufs paraissant avoir la taille la plus communément observée, on arrive comme moyenne à $59 \mu$ sur $34 \mu$. Les chiffres donnés par les auteurs japonais sont un peu plus forts. Yoshida (1917) indique 62 à $76,9 \mu$ sur 33 à $43,5 \mu$, soit, en calculant la moyenne, 67,2 sur 37,93 . Yamata (1917) indique 68,5 sur 36,3 . Nous pensons que ces variations n'ont pas une très grande importance; elles ont déjà été notées pour plusieurs espèces de Diphyllobothrium : D. latum, D. decipiens ; Yoshida et Kotlan les ont étudiées. La taille des œufs paraît varier avec l'hôte du ver, probablement aussi avec d'autres facteurs. Dans un même anneau, il n'existe aucune différence de taille appréciable entre les œufs qui se trouvent au début de l'utérus et ceux qui sont près de l'orifice de ponte; ils ne grandissent done pas dans le tube utérin.

IDENTIFICATION DE NOTRE BOTHRIOCÉPHALE. - C'est incontestablement un Diphyllobothrium. Malheureusement le diagnostic des diverses espèces de ce genre est loin d'être facile. Leur structure anatomique ne présente que de faibles variations, d'où difficulté de 
les différencier les unes des autres. De plus, nous avons vu que des caractères commodes à apprécier, comme la taille des œufs, varient dans la même espèce ; nous avons constaté également que la disposition des testicules et des vitellogènes peut, au moins dans le cestode que nous avons étudié, n'être pas toujours uniforme chez le même ver. Enfin les bothriocéphales signalés par les auteurs anciens, et même par quelques auteurs modernes, sont trop souvent décrits d'une façon sommaire.

Nous estimons qu'il y a lieu de négliger et de considérer comme nomina nuda les espèces mal décrites, dont la diagnose est impossible à vérifier. Nous renvoyons à la revue critique de Baer (1925, p. 13-16) et n'admettons que les espèces conservées par cet auteur dans son tableau récapitulatif des Diphyllobothrium de mammifères, ainsi que celles parues depuis.

Nous pensons également devoir faire intervenir la notion biologique. Il est évident que la répartition géographique de Sparganum mansoni cadre avec celle du bothriocéphale adulte lui correspondant. Nous pouvons done éliminer de ce fait les Diphyllobothrium décrits chez les pinnipèdes et les cétacés des régions arctiques.

Avec cette double restriction, il nous reste à discuter les espèces suivantes.

D. minor Cholodkovsky, 1916, de l'homme, en Sibérie, se distingue de notre espèce par sa longueur plus petite (100 millimètres) et ses œufs de taille plus consiđérable $(70 / 40 \mu)$.

D. trnioides (Leon 1916), de l'homme, en Roumanie, est considéré par Baer comme une espèce douteuse, peut-être comme une malformation de $D$. latum. En tous cas, il est insuffisamment décrit pour être discuté.

D. serratum (Dies., 1850) des Canida brésiliens diffère de notre espèce par sa taille plus grande $(1 \mathrm{~m} ., 900 / 0 \mathrm{~m}$., 020$)$ et les dimensions de ses œufs $(25 \mu)$, ainsi que par sa répartition géographique.

D. bresslaui Baer, 1927, de Didelphys marsupialis, au Brésil, a une taille différente (270 mill./8 mill.) ; il se distingue aussi par son hôte et sa répartition géographique.

D. gracile Baer, 1927, d'Oncoides weidi, au Brésil, se différencie aussi par sa répartition géographique (1).

D. tangalongi (Mac Callum, 1921) de Viverra tungalunga, à Bornéo, est insuffisamment connu, d'après Baer, pour que l'on soit fixé

(1) D. bresslani et D. gracile portent, dans des travaux antérieurs, la date de 1924. En réalité, le manuscrit de J.-G. Baer, décrivant ces espèces, remis à l'imprimerie en 1924, ne doit paraitre qu'en 1927. 
sur sa validité. Le scolex figuré et décrit par Mac Callum (1921, p. 216) ne paraît pas semblable à celui de nos échantillons. Chaque anneau recouvre très largement le suivant, ils sont en forme de cloche. En tous cas, les œufs, mesurant $40 \mu$ sur $20 \mu$, sont plus petits que ceux de notre espèce.

D. sulcatum (Molin, 1858), de Leopardus pardus de l'Inde, est aussi assez mal connu. Il mesure $1 \mathrm{~m}$., 300 sur $0 \mathrm{~m}$., 006. Ses œufs ont $62 \mu$ sur $37 \mu$. D'après Ariola (1900, p. 422), cette espèce serait caractérisée par la position des bothridies : " questa specie, per la posizione dei botridii sui margini, resta ben distincta da tutte le altre parassite dei felini, le quali sono caratterizate dallo avere le fossette sulle facce piane dello scolice ".

D. latum (L., 1758) des carnivores et de l'homme, cosmopolite, se distingue facilement de notre espèce. Sa taille est plus grande, ses bothridies à bord flottant moins développé ; ses testicules et ses vitellogènes ne se rejoignent jamais à la partie supérieure de l'anneau ; ils sont toujours disposés sur deux champs latéraux, l'utérus forme une rosette caractéristique autour de l'orifice de ponte, les œufs mesurent $70 \mu$ sur $45 \mu$.

D. theileri Baer, 1925, décrit chez les félidés de l'Afrique du sud, possède un atrium génital bien marqué. L'orifice de ponte est souvent déplacé par rapport au pore génital, il alterne à droite et à gauche avec ce dernier. Il existe une vésicule séminale interne, le diamètre des vaisseaux aquifères n'atteint que $11 \mu$.

Diphyllobothrium pretoriense (Baer, 1924) (=Lüheella pretoriensis Baer, 1924)(1), trouvé en Afrique du sud chez le carnivore Otocyon megalotis Desm., diffère de notre cestode par plusieurs caractères. Les testicules sont répartis sur deux champs latéraux ne se réunissant pas à la partie antérieure. L'utérus présente une boucle très caractéristique, fortement dilatée, se trouvant tantôt à droite, tantôt à gauche de la ligne médiane. Sa portion terminale est fortement musclée et montre de très nombreuses glandes qui manquent dans notre bothriocéphale.

D. raillieti (Ratz, 1892), du chien de Serbie et de Hongrie, est

(1) Note additionnelle de J.-G. Baer el Ch. Joyeux. L'un de nous (Baer, 1924, note préliminaire, et 1925 loc. cit.) a créé pour ce bothriocéphale un nouveau genre Lüheella ct une nouvelle famille Lüheellida, basés sur l'absence de vésicule séminale externe, caractère primordial de la famille des Diphyllobotriida Lühe, 1910. L'étude de la poche du cirre chez $D$. mansoni, $D$. railliefi et $D$. theileri nous a montré tous les types intermédiaires entre celle de $D$. latum d'une part et de L. pretoriensis d'autre part. Pour ne pas surcharger la nomenclature, nous estimons maintenant que la création d'une nouvelle famille et, par suite, d'un nouveau genre pour désigner ce cestode n'est pas justifiée. En conséquence nous faisons tomber en synonymie la famille des Luheellida avec celle des Diphyllobothriida et le genre Lüheella avec Diphyllobothrium. L'espèce en question devient donc : Diphyllobothrium pretoriense (Baer, 1924). 
très semblable comme morphologie à notre espèce, d'après la description de Kotlan (1923). Les œufs mesurent 62 à $72 \mu$ sur 37 à $45 \mu$. Les testicules sont disposés sur deux champs latéraux, ne se joignant pas à la partie supérieure de l'anneau : " In dem Mittelfelde kommen sir nicht vor ". Toutefois Kotlan ne dit pas si cette disposition a été vérifiée sur quelques anneaux seulement ou sur une notable partie de la chaîne ; d'après ce que nous savons de notre espèce, nous pensons que ce caractère doit être observé sur un grand nombre d'anneaux avant de tirer une conclusion. Néanmoins, pour des raisons biologiques, nous distinguons notre espèce de $D$. raillieti. Le plérocercoïde de celui-ci : Sparganum raillieti, s'observe seulement chez le porc en Serbie et en Hongrie, tandis que Sparganum mansoni se voit chez toutes sortes de vertébrés, en Extrême-Orient; il est donc plus ubiquiste et doit, à notre avis, correspondre à une autre espèce.

D. reptans (Dies., 1850). La larve plérocercoïde de ce bothriocéphale a été décrite par Diesing (Ligula reptans Dies., 1850, p. 581); il la signale chez de nombreux vertébrés : mammifères, oiseaux, reptiles et amphibiens de l'Amérique du sud. Meggitt (1924), à Rangoon (Birmanie), a retrouvé un Sparganum qu'il assimile à $S$. reptans, commun chez les reptiles de ce pays. Il a fait développer ce plérocercoïde, obtenu d'une couleuvre : Tropidonotus sp., chez le chien, mais n'a pu réussir chez l'homme. Le Diphyllobothrium reptans, obtenu ainsi expérimentalement, a été décrit par Meggitt. Grâce à l'amabilité de cet auteur, nous en possédons des cotypes, ce qui nous facilite la comparaison avec notre ver; de plus, nous avons retrouvé D. reptans en Indochine, comme nous le verrons ci-dessous. Le nombre des testicules atteint seulement 144 à 200 par anneau, d'après le texte de Meggitt. En réalité, ces chiffres nous paraissent un peu faibles, l'examen du matériel cotype nous ayant donné une moyenne de 270 testicules par anneau. Ils sont placés sur deux champs latéraux, se rapprochant à la partie antérieure. Meggitt indique et figure ce caractère ; nous l'avons également vérifié sur 94 anneaux, provenant des types de cet auteur. Les vitellogènes peuvent aussi se rapprocher sans se toucher; nous reviendrons plus loin sur cette disposition. La poche du cirre se trouve presque au niveau du bord supérieur de l'anneau (dans le 1/6 d'après Meggitt). Enfin l'orifice femelle est déplacé sur le côté, par rapport aux orifices mâle et utérin qui sont sur la ligne médiane. Nous dirons qu'on les trouve aussi quelquefois tous trois en ligne droite, mais c'est rare. Enfin, au point de vue biologique, nous avons dit que, contrairement aux données anciennes de Diesing, Meggitt estime que la larve plérocercoîde : Sparganum reptans, se trouve 
sans doute exclusivement chez les reptiles, tandis que celle de notre espèce est hébergée par de nombreux vertébrés de toutes classes. Nous concluons done que $D$. reptans, très voisin de $D$. mansoni, s'en distingue par de légères différences morphologiques et par l'habitat de la larve plérocercoïde.

Diphyllobothrium ranarum (Gastaldi, 1854) a été obtenu expérimentalement chez le chien par Meggitt (1925), en partant d'un Sparganum de grenouille de Birmanie. Cet auteur l'a récemment retrouvé dans la nature, également chez le chien (1927). Il pense que sa première expérience est peut-être entachée d'erreur. Quoi qu'il en soit, D. ranarum est différent de D. mansoni. Il paraît très voisin de D. reptans, sinon identique à lui. J.-G. Baer et l'un de nous donneront prochainement une étude plus approfondie de cette espèce, après examen des catypes de Meggitt.

D. decipiens (Diesing, 1850) a été signalé par Diesing (Dibothrium decipiens Diesing, 1850 , p. 588) chez une série de félidés surtout brésiliens. La première description est courte. Les exemplaires. existant au musée de Vienne, ont été revus par Lühe (1899), qui a distingué de ce ver le $D$. latum par l'absence de rosette utérine autour de l'orifice de ponte. Ariola (1900), Böhm (1923) en ont donné des descriptions incomplètes. Chandler l'a retrouvé à Calcutta chez le chat (1 fois sur 200) et chez Felis nebulosa (1925). Actuellement on le connait chez de nombreux carnivores. Nous avons pu en examiner quelques anneaux, provenant des types du musée de Vienne, offerts autrefois au professeur Blanchard. Nous avons dit que Ando et Kobayashi pensent que D. mansoni se confond avec D. decipiens; cependant nous trouvons les caractères différentiels suivants. Les testicules et les vitellogènes se joignent toujours à la partie antérieure de l'anneau. Chandler montre cette disposition dans sa figure de D. decipiens (pl. V, fig. 1) et spécifie dans son texte : "The yolk glands and testes are evenly scattered throughout the lateral portion of the segments, and across the middle portion anteriorly, but leave a conspicuous clear centra! area surrounding the uterus and genital openings. " Nous n'avons pu vérifier ce caractère que sur quatre anneaux provenant du musée de Vienne, n'ayant pas eu de matériel plus abondant à notre disposition. Jusqu'à nouvelle indication,. nous admettons donc l'exactitude du caractère donné par Chandler, différenciant $D$. decipiens de notre espèce.

Chez $D$. decipiens, l'orifice femelle est plus rapproché de l'orifice utérin que de l'orifice mâle. Nous avons vérifié ce caractère, donné par Chandler, sur nos types du musée de Vienne. Dans les quelques anneaux que nous avons examinés, le cirre est évaginé et son extrémité est introduite dans le vagin, les deux orifices sont donc faciles 
à repérer. En prenant la moyenne de nos mensurations, nous arrivons aux chiffres suivants :

Distance du bord antérieur de l'anneau au milieu de l'orifice mâle.................................

Distance du milieu de l'orifice màle au milieu de l'orifice femelle.....................................

Distance du milieu de l'orifice femelle au milieu de l'orifice utérin ....................................

Distance du milieu de l'orifice utérin au bord postérieur de l'anneau.................................. $2 \mathrm{~mm}, 400$

Longueur de l'anneau.................. $\quad \overrightarrow{3 \mathrm{~mm} ., 680}$

$0 \mathrm{~mm} ., 800$

$0 \mathrm{~mm} ., 310$

$0 \mathrm{~mm} ., 170$

Nous avons vu que chez notre ver, contrairement à ce qui s'observe chez $D$. decipiens, l'orifice femelle est toujours plus rapproché de l'orifice mâle que de l'orifice utérin.

Les boucles utérines, chez $D$. decipiens, vont en augmentant d'ampleur d'arrière en avant, c'est-à-dire de la naissance à la terminaison de cet organe. Nous avons également vérifié ce caractère, donné par Chandler, sur les types du musée de Vienne. Chez notre ver, on observe le contraire ; nous avons dit qu'il existe trois boucles qui vont en diminuant à mesure qu'on approche de l'orifice de ponte.

Les œufs de $D$. decipiens mesurent 60 à $68 \mu$ sur 36 à $40 \mu$, ils sont donc un peu plus grands que ceux de notre espèce, mais nous avons déjà fait observer que ce caractère différentiel est assez médiocre.

Enfin il nous semble que la biologie peut servir aussi à différencier ces deux bothriocéphales. Alors que Sparganum mansoni est fréquent en Extrême-Orient chez de nombreux vertébrés, dont l'homme, le plérocercoïde de $D$. decipiens parait avoir une spécificité parasitaire plus étroite. Nous avons dit que Diesing l'a décrit surtout chez des félidés brésiliens; or les Sparganum sont moins souvent signalés au Brésil qu'en Extrême-Orient; la sparganose humaine, notamment, y paraît inconnue.

En résumé, le bothriocéphale adulte, correspondant à Sparganum mansoni, se distingue aussi bien par ses caractères morphologiques que biologiques des espèces connues de Diphyllobothrium; en ne tenant compte que de celles dont la description est suffisante pour permettre une comparaison, les autres devant, à notre avis, être considérées comme nomina nuda. Il mérite done de conserver son nom spécifique, qui est, d’après les règles de la nomenclature : Diphyllobothrium mansoni (Cobbold, 1883).

Diagnose de D. mansoni. - Caractères généraux du genre Diphyllobothrium. Dimensions : 60 centimètres à 1 mètre sur 5 à 6 milli- 
mètres. Anneaux plus larges que longs, excepté dans la portion terminale de la chaîne, où la longueur finit par égaler à peu près la largeur. Partie postérieure de chaque anneau débordant légèrement le suivant. Scolex mesurant $1 \mathrm{~mm}$. à $1 \mathrm{~mm}$., 5 sur $0 \mathrm{~mm}$., 4 à $0 \mathrm{~mm}$., 8, presque quadrangulaire ; bothridies à bords flottants développés. Testicules et vitellogènes sur deux champs latéraux, pouvant ou non se joindre à la partie antérieure de l'anneau. Testicules variant en nombre de 380 à 540 par anneau, généralement disposés en une seule rangée transversale, ayant 135 à $160 \mu$ de diamètre. Utérus décrivant trois boucles d'amplitude décroissante en se rapprochant de l'orifice de ponte. Extrémité de l'utérus non bourrée d'œufs comme le reste de l'organe. Pas de rosette autour de l'orifice de ponte. Orifice femelle plus rapproché du mâle que de l'utérin ; tous trois en ligne droite. CEufs de dimensions variables : 52 à $62 \mu$ sur 32 à $40 \mu$; moyenne : $59 \mu$ sur $34 \mu$, d'après nos mensurations. 62 à 76,9 sur 33 à 43,5; moyenne : 67,2 sur 37,93 d'après Yoshida ; 68,5 sur 36,3 d'après Yamata.

Hôte de l'adulte (expérimental) : chien, chat (1).

Hôte du procercoïde : Cyclops leuckarti Sars., d'après Okumura.

Hôte du plérocercoïde : nombreux vertébrés, dont l'homme (mammifères, oiseaux, reptiles, batraciens).

Diphyllobothrium reptans (Dies., 1850). - Dans l'intestin du chat domestique et de Zibethailurus nebulosus Griffith, à Hanoï (2). Ce ver existe également dans les collections du laboratoire de parasitologie, recueilli chez le chien par le $\mathrm{D}^{r}$ Gauducheau. Nos exemplaires provenant de félidés sont jeunes; le plus grand ne mesure que 27 centimètres ; nous croyons pouvoir les déterminer d'après la description de Meggitt (1924) et les types que cet auteur a bien voulu nous envoyer.

Les œufs de nos vers mesurent 53 à $57 \mu$ sur 27 à $28 \mu$ (53 à $59 \mu$ sur 36 à $40 \mu$ d'après Meggitt). L'orifice vaginal se trouve sur le côté, par rapport aux orifices mâle et utérin qui sont sur la ligne médiane, comme l'indique bien Meggitt dans son texte. Sur quelques

(1) Baer (1925, p. 16), indique Thos aureus comme hôte de D. mansoni. Il y a probablement confusion entre l'hôte définitif et l'hôte du plérocercoïde. Sonsino (1889 a p. 232) décrit, en effet, un Sparganum qu'il identifie, d'après les travaux de Leuckart, à $S$. mansoni ; ce parasite avait été trouvé au Caire par Walter Innés, dans le tissu.sous-cutané d'un chacal, que Sonsino nomme "Canis aureus ".

(2) A Saïgon, nous avons trouvé le pourcentage suivant de bothriocéphales : 11,68 chez le chien et 14,17 chez le chat.

Quelques uns de ces bothriocéphales provenant du chat ont été déterminés ; il s'agissait toujours de $D$. reptans, mais il est possible que d'autres espèces soient aussi hébergées par cet animal. Pour ce qui est du chien, aucun bothriocéphale n'a été examiné : il aurait été intéressant de savoir si ce carnivore qui est facilement infesté expérimentalement par $D$. mansoni, héberge ce parasite dans la nature et si c'est l'hôte normal du ver adulte. 
anneaux, cependant, ils paraissent tous trois en ligne droite, aussi bien dans le type que dans nos exemplaires. Les testicules sont sur deux champs séparés, convergeant légèrement à la partie antérieure de l'anneau ; dans nos échantillons, ils convergent plus ou moins. Pour les vitellogènes, Meggitt indique qu'ils convergent et se rencontrent à la partie antérieure de l'anneau : "Vitelline glands lateral, converging and meeting anteriorly "; mais il arrive aussi que les vitellogènes ne se rejoignent pas ; cette disposition s'observe dans nos exemplaires et dans les types de Meggitt. Cet auteur l'a certainement notée aussi, puisqu'elle est représentée dans sa figure (pl. IX, fig. 2).

Pour le reste de la description, nous ne voyons aucune différence.

\section{TETRAPHYLLIDEA}

\section{Ichthyotaeniidæ}

Ichthyotænia naiæ (Beddard, 1913)._- Trouvé chez Bungarus fasciatus Schneid. (ophidien), environs de Hanoï. Notre cestode correspond à la description de Woodland (1925); seule la poche du cirre est un peu moins longue et un peu plus large, elle mesure 450 à $480 \mu$ sur $150 \mu$ de large (Woodland donne 498 à $531 \mu$ sur 83 à $107 \mu) .1$. naiæ a été décrit chez Naja tripudians Mer., voisin de Bungarus fasciatus tant au point de vue zoologique qu'à celui de la répartition géographique. Meggitt a récemment démontré $(1927, a)$ que le terme Ichthyotænia a la priorité sur Proteocephalus.

\section{Deuxième partie. Trématodes}

\section{A MPHISTOMATA}

\section{Paramphistomidæ}

Paramphistomum explanatum (Creplin, 1847). - Commun dans les canaux biliaires du bœuf et du buffle.

Homalogaster paloniæ Poirier, 1883, Syn. H. poirieri Giard et Billet, 1892. - Intestin du bœuf dans la proportion de 47,05 p. 100 , de la chèvre dans celle de 5 p. 100 , à Hanoï.

\section{DISTOMATA}

\section{Fasciolidæ}

Fasciola hepatica L., 1758. - Nous avons récolté la grande douve hépatique chez les animaux suivants : bovidés (bœuf et buffle) : 
64,7 p. 100 des sujets examinés ; ovidés : 52,94 p. 100 ; capridés : 35 p. 100 ; lapin domestique : 14,28 p. 100.

Elle existe également chez le pore, avec perforation du foie dans . un cas, le cobaye et divers cervidés sauvages.

Fasciola gigantica (Cobbold, 1856). - Dans le foie des bovidés à Hanoï (proportion de 23,52 p. 100) et à Nha'Trang (récolté dans cette dernière localité par le $\mathrm{D}^{r}$ Lagrange).

Fasciolopsis buski (Lankester, 1857). - Intestin du porc, à Hanoï. 73,04 p. 100 des pores sacrifiés à l'abattoir municipal de Hanoï en sont porteurs.

\section{Opisthorchiidæ}

Clonorchis sinensis (Cobbold, 1875). - Foie du chien (10,94 p. 100) et du chat $(32,83$ p. 100$)$, à Hanoï.

\section{Echinostomidæ}

Echinochasmus perfoliatus (Ratz, 1908):- Intestin du chien, à Hanoï (proportion de 0,29 p. 100).

\section{Philophthalmidæ}

Philophthalmus gralli Mathis et Leger, 1910, - Culs-de-sac conjonctivaux de la poule $(1,21$ p. 100$)$, du canard $(7,69$ p. 100), de l'oie (5 p. 100) ; trouvé également chez le paon : Pavo muticus Horsf.

\section{Lepodermatidæ-Plagiorchidæ}

Prosthogonimus brauni (Skriabine, 1919). - Cloaque de la poule domestique $(19,39$ p. 100$)$; observé une fois dans l'oviducte.

Nous n'avons déterminé qu'un petit nombre d'exemplaires qui cadrent avec la description de Skriabine, excepté cependant pour ce qui concerne la bifurcation des branches intestinales. Chez $P$. brauni, elle se fait immédiatement en avant de la ventouse ventrale; chez $P$. pellucidus (Linst., 1873), elle est beaucoup plus antérieure, le dessin de Braun (1902, pl. IV, fig. $45 a$ ) la représente à égale distance du bord antérieur de la ventouse ventrale et du bord postérieur de la ventouse orale. Dans nos échantillons, nous comptons $450 \mu \mathrm{du}$ bord antérieur de la ventouse ventrale à la bifurcation, $600 \mu$ de la bifurcation au bord postérieur de la ventouse orale; ils sont donc intermédiaires entre la disposition de $P$. brauni et celle de $P$. pellucidus. Les anses utérines sont du type $P$. brauni, plus nombreuses que chez $P$. pellucidus et régulièrement ordonnées.

A. Railliet a décrit (1924) Prosthogonimus furcifer, récolté par 
Bauche à Hué (Annam), dans la bourse de Fabricius d'un poulet. Malheureusement, par suite des circonstances, cette description n'a pu être donnée complètement. De plus, la difficulté de se procurer les publications de langue russe au moment où le professeur A. Railliet étudiait son matériel l'a empêché de prendre connaissance du travail de Skriabine. Nous-mêmes n'avons en notre possession qu'un mémoire plus récent (1923), qui reproduit heureusement la diagnose originale avec une figure. Il est possible que Prosthogonimus furcifer Railliet, 1924 tombe en synonymie avec $P$. brauni Skriabine, 1919. Le nombre insuffisant de nos exemplaires ne nous permet pas de trancher cette question.

Prosthogonimus cuneatus (Rud., 1819). - Cloaque de Corvus torquatus Lesson. Région de Hanoï.

Un seul échantillon, cadrant avec les descriptions des auteurs (Braun, 1902, Skriabine, 1913).

Prosthogonimus rudolphii Skriabine, 1919. - Bourse de Fabricius du canard domestique, Hanoï.

Nous n'avons qu'un seul exemplaire jeune, ne montrant pas encore d'œufs dans l'utérus. Les branches intestinales sont un peu plus longues que dans le dessin de Skriabine (1923) et atteignent une boucle de l'utérus qui les croise. L'ovaire est aussi plus rapproché de la ventouse ventrale.

Encyclometra caudata (Polonio, 1859). - Intestin de Zamenis mucosus (L.) (Ophidien), Tonkin.

Ce trématode a été récemment décrit sous de nouveaux noms : Odhneria bolognensis Baer, 1924 ; Encyclometra natricis Baylis et Cannon, 1924. Il a été ultérieurement identifié par R.-Ph. Dollfus (1924), à Encyclometra subflava (Sonsino, 1892). Depuis la publication de son travail, R.-Ph. Dollfus a eu l'occasion d'étudier à nouveau la synonymie de cette espèce et il nous communique la note suivante, encore inédite. "Le nom à adopter est Encyclometra caudata (Polonio, 1859). Ce nom spécifique, proposé par Polonio, est plus ancien que celui de Sonsino. Selon toute vraisemblance, le Distomum colubrimurorum Rud., $1819(=D$. allostomum Dies., 1850, nom., nov.), concerne la même espèce, mais le type de Rudolphi, ainsi que nous l'a écrit le $\mathrm{D}^{r}$ Collin, n'existe plus dans la collection de Berlin ; il est donc impossible de savoir, si, au lieu d'E. caudata (Polonio), il aurait fallu adopter Encyclometra colubrimurorum (Rud.). »

Ce trématode, des couleuvres de la région méditerranéenne, s'étend done jusqu'à l'Extrême-Orient. Il a d'ailleurs déjà été signalé en Birmanie par Bhalerao (1926). 
Glyphtelmis linguatula (Rud., 1819). - Intestin de Rana tigrina Daud., Tonkin.

Nous basons notre détermination sur la description récente de L. Travassos (1924), à laquelle correspondent nos échantillons.

Styphlodora lachesidis Mac Callum, 1921. - Intestin de Zamenis mucosus L. (ophidien).

Nos exemplaires correspondent à la description de Mac Callum (1921, p. 158-159).

\section{Lecithodendriidæ}

Lecithodendrium sp. - Duodénum de Mabuia siamensis Gunther (Saurien), Tonkin.

Un seul exemplaire abimé, difficilement déterminable.

Prosotocus confusus (Looss, 1894). - Intestin (région pylorique) de Rana tigrina Daud, Tonkin.

Nos échantillons concordent avec les descriptions des auteurs ; notons la grande taille des ventouses : 180 à $200 \mu$ pour la ventouse orale, 160 à $180 \mu$ pour ventouse ventrale.

\section{Dicrocœliidæ}

Eurytrema pancreaticum (Janson, 1889). - Canaux biliaires du bouf (29,4 p. 100), du buffle, du mouton, de la chèvre (15 p. 100). La taille de ce trématode est moindre chez les petits ruminants que chez les grands. Déjà souvent signalé en Indochine.

Paradistomum mutabile (Molin, 1859). _ Vésicule biliaire de Mabuia siamensis Gunther (saurien), Tonkin.

Deux échantillons. Nous renvoyons, pour l'histoire et la morphologie de cette espèce au travail de R.-Ph. Dollfus (1922-1923). Nos échantillons diffèrent de $P$. mutabile type parce que les vitellogènes s'étendent au-delà du bord antérieur des testicules ; cependant ils sont loin d'atteindre le pore génital comme chez Paradistoma ceratophoræ (Looss, 1908), R.-Ph. Dollfus, 1923. Nous pensons qu'il s'agit simplement d'une variation de $P$. mutabile.

\section{Clinostomidæ}

Ithyoclinostomum heterostomum (Rud., 1809). - Chez l'aigrette, Garzetta garzetta Sharpe.

Le genre Ithyoclinostomum a été créé par Witenberg (1925, p. 175, en note) pour cette espèce.

Nos échantillons concordent avec la description de Braun (1900), en particulier le tube digestif à diverticules typiques (fig. 12). Cette espèce paraît cosmopolite. 
L'histoire épidémiologique de ce trématode est intéressante. Il a été récolté en Cochinchine, chez des aigrettes élevées en vue de la récolte des plumes. Le parasite se fixe sur les muqueuses buccale et pharyngienne, gênant à la fois la déglutition et la respiration ; on peut le trouver autour de la glotte. Il provoque la mort de l'oiseau par asphyxie et par inanition.

On sait que plusieurs espèces de Clinostomum se trouvent chez des poissons à l'état de métacercaires. Précisément, les aigrettes de l'élevage en question sont nourries avec des Anabas scandens Dald. Nous avons pu examiner quelques-uns de ces poissons; leurs muscles contenaient de nombreuses métacercaires ; malheureusement le matériel mal conservé ne nous a pas permis d'étaler ces larves et nous n'avons, par suite, aucune donnée sur leur correspondance avec la forme adulte observée.

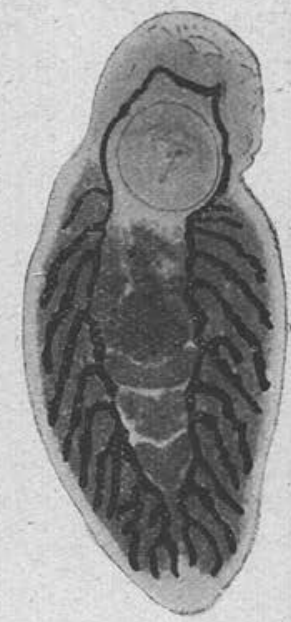

FIG. 12. - Ithyoclinostomum helerostomum (Rud.) Microphotographie montrant l'aspect des branches intestinales. Le tube digestif de cet exemplaire s'est trouvé naturellement injecté en noir.

\section{Harmostomidæ}

Harmostomum (Postharmostomum) commutatum (Dies., 1858). - Cæcums du dindon domestique, Hanoï. (Proportion : 9,09 p. 100).

Witenberg, en 1923 (in 1925, texte allemand), a subdivisé le genre Harmostomum Braun, 1899 en deux sous-genres : Harmostomum (Harmostomum) et Harmostomum (Postharmostomum). C'est à ce dernier qu'appartient notre espèce. Cet auteur a créé, en même temps, une espèce nouvelle: Harmostomum (Postharmostomum) gallinum Wit., 1923, qu'il a distingué de Harmostomum (Postharmostomum) commutatum (Dies., 1858) ; toutes deux parasites des cæcums de divers oiseaux.

H. commutatum a été déerit par Wagener (1852), sous le nom de Distoma dimorphum (nec $D$. dimorphum Dies., $1839=$ Clinostomum). A cause de cette priorité, l'espèce de Wagener a été nommée à nouveau par Diesing : D. commutatum Dies., 1858. Wagener l'a récoltée chez la poule, à Pise et à Nice; il en a donné une description et une bonne figure. Le parasite a été étudié à nouveau par Sonsino (1889 b, p. 282) qui l'a aussi trouvé à Pise, chez la poule, et rapporte à ce parasite un jeune distome du dindon (1891). 
Galli-Valerio et peut-être Stossich l'auraient également signalé, d'après Witenberg.

H. gallinum a été trouvé par Witenberg chez la poule du Turkestan russe. L'un de nous (Joyeux, 1923) a décrit, en Tunisie, avec figure à l'appui, un trématode qu'il a assimilé à $H$. commutatum, les travaux de Witenberg n'étant pas encore connus à cette époque. Ce trématode a été recueilli en assez grande abondance chez une poule, à Madhia (Tunisie) ; un autre exemplaire, unique, a été récolté chez une poule vendue au marché de Tunis; nous l'avons également vu une fois chez la pintade [Numida (Numida) meleagris L.] ; enfin nous croyons pouvoir rapporter à cette espèce un jeune ver trouvé chez le pigeon domestique.

Witenberg, d'après l'examen de notre dessin, a rangé nos trématodes dans sa nouvelle espèce : $H$. $(\boldsymbol{P}$.) gallinum.

D'après cet auteur, les différences entre $H$. commutatum et H. gallinum sont les suivantes :

$1^{\circ}$ Vitellogènes plus développés chez $H$. commutatum, leur extrémité antérieure atteignant la bifurcation intestinale.

$2^{\circ}$ Ventouse orale arrondie chez $H$. commutatum, ovale à grand axe transversal chez $H$. gallinum.

$3^{\circ}$ Pore génital au niveau du bord antérieur du testicule antérieur chez $H$. commutatum ; en arrière de ce bord chez $H$. gallinum.

$4^{\circ}$ Branches intestinales plus sinueuses chez $H$. gallinum.

Après révision de nos échantillons de Tunisie, nous allons diseuter ces divers caractères (voir fig. 13 et 14 ).

$1^{\circ}$ Sur tous nos échantillons, nous observons le caractère mentionné par Witenberg: l'extrémité antérieure des vitellogènes s'avance au plus jusqu'au niveau du bord postérieur de la ventouse ventrale, rarement le déborde légèrement. On remarquera toutefois que les vitellogènes ne s'arrêtent pas toujours à un niveau fixe, mais que leur extrémité antérieure varie d'un exemplaire à l'autre, et même de droite à gauche chez un seul ver. D'autre part, la figure de Wagener représente bien ces organes s'avançant jusqu'au niveau de la bifurcation intestinale ; Sonsino dit qu'ils occupent le tiers moyen et antérieur.

$2^{\circ}$ La ventouse orale est, sur nos échantillons, tantôt arrondie, tantôt ovalaire; on observe toutes les transitions entre ces deux positions extrêmes.

$3^{\circ}$ Le pore génital est figuré par Wagener en avant du testicule antérieur. Sonsino dit aussi qu'il est placé " davanti il testicolo anteriore ". Dans notre figure de 1923 , nous l'avons dessiné un peu en arrière du testicule. En réalité, nos échantillons montrent 
divers types de pores génitaux : en avant du testicule antérieur, latéral à cet organe, avec toutes les positions intermédiaires.
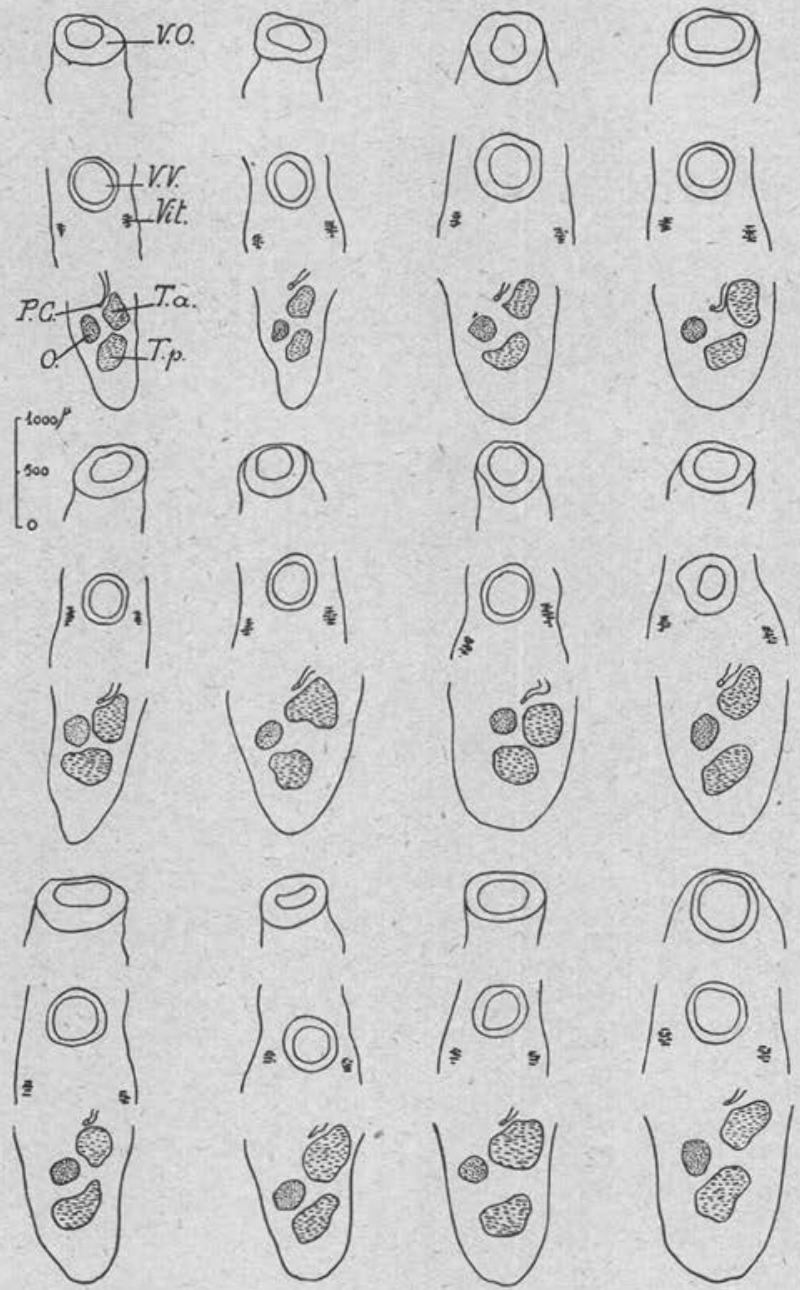

Fig. 13. - Schéma de 12 Harmostomum (Postharmostomum) commutatum (Dies.) indiquant la forme de la ventouse orale, l'extrémité antérieure des vitellogènes, la position du pore génital par rapport au testicule antérieur. VO, ventouse orale; $V_{v}$, ventouse ventrale; Vit, extrémité antérieure des vitellogènes; $P G$, pore génital; Ta, testicule antérieur; Tp, testicule postérieur; 0 , ovaire.

$4^{\circ}$ Les branches intestinales, sur nos échantillons, sont plus ou moins coudées, suivant l'état de contraction de l'animal. 
Nous ne trouvons aucune différence entre nos types et ceux décrits par Wagener et Sonsino. Nos œufs mesurent 27 à $29 \mu$ sur 13 à $14 \mu$; Sonsino donne $27 \mu$ sur $14 \mu$; Witenberg, pour son H. gallinum, 29 à $32 \mu / 18 \mu$.

Enfin, au point de vue biologique, les exemplaires de Wagener et Sonsino ont été récoltés à Nice et à Pise, les nôtres dans le nord de la Tunisie ; ils proviennent done tous d'une même région méditerranéenne, dans laquelle les échanges économiques sont actifs, ce qui explique facilement le transport des parasites d'un point à un autre.

En résumé, un seul caractère reste pour distinguer $H$. commuta-
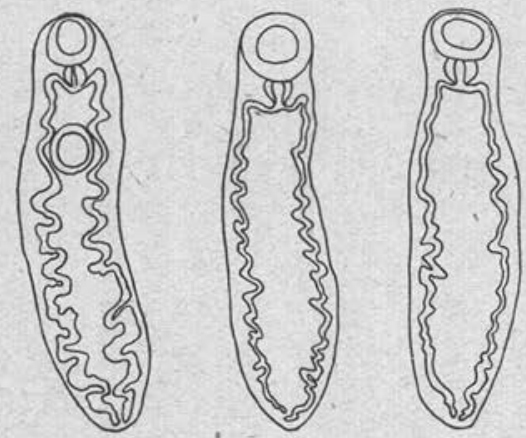

Fig. 14. - Schéma de 3 Harmostomum (Postharmostomum) commutatum, indiquant les variations de forme des branches intestinales.

tum de $H$. gallinum : les rapports de l'extrémité antérieure des vitellogènes. On sait, d'autre part, que ces organes présentent souvent des modifications assez marquées chez les trématodes et il nous semble excessif de fonder une espèce basée exclusivement sur ce caractère. Nous pensons donc qu'Harmostomum (Postharmostomum) gallinum Wit. n'est qu'une simple variation d'Harmostomum (Postharmostomum) commutatum (Dies.).

Harmostomum (Postharmostomum) gallinum tombant en synonymie avec Harmostomum (Postharmostomum) commutatum, il s'ensuit que le type de ce sous-genre, qui était gallinum, devient commutatum. Il aurait dû l'être d'ailleurs dès la création du sous.genre Postharmostomum, comme plus ancien que gallinum.

Nous avons donc : sous-genre Postharmostomum Witenberg, 1923. Espèce type: Harmostomum (Postharmostomum) commutatum (Wagener, 1852). 


\section{Schistosomatidæ}

Schistosoma spindale Montgomery, 1906. - Système porte du bœuf et du buffle. Trouvé par le $\mathrm{D}^{r}$ Lagrange à Nha'Trang et par nous à Hanoï. Ce parasite est déjà connu en Indochine.

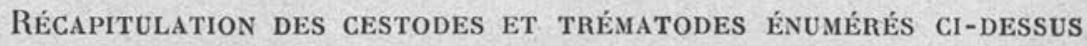

CLASSÉS D'APRĖS LEURS HOTES

\section{Mammifères}

Homme. Trnia saginata. Sparganum mansoni.

Macacus cynomolgus. Bertiella studeri. Sparganum mansoni. Tinia hydatigena.

Macacus sinicus. Bertiella studeri.

Macacus nemestrinus. Sparganum mansoni.

Semnopithecus sp. Cysticercus tenuicollis.

Nemorrhaeus maritimus. Cysticercus tenuicollis.

Porc domestique. Echinococcus granulosus (larve). Cyslicercus lenuicollis. Cyslicercus cellulosæ. Fasciola hepatica. Fasciolopsis buski.

Bœuf domestique. Cysticercus bovis. Echinococcus granulosus (larve). Homalogaster paloniz. Paramphistomum explanatum. Fasciola hepatica. Fasciola gigantica. Eurytrema pancreaticum. Schistosoma spindale.

Buffle domestique. Homalogaster polonix. Paramphistomum explanalum. Fasciola hepatica. Fasciola gigantica. Eurytrema pancreaticum. Schistosoma spindale.

. Mouton domestique. Fasciola hepatica. Cysticercus tenuicollis. Eurytrema pancreaticum.

Chèvre domestique. Cysticercus tenuicollis. Homalogaster paloniæ. Fasciola hepatica. Eurytrema pancreaticum.

Cervides sp. Fasciola hepatica.

Lapin domestique. Fasciola hepatica.

Epimys norvegicus. Sparganum mansoni.

Epimys rattus. Sparganum mansoni.

Rat sp. Raillietina (Raillietina) celebensis. Cỳslicercus fasciolaris.

Cobaye. Fasciola hepatica.

Chien domestique. Trenia pisiformis. Trenia hydatigena. Dipylidium caninum. Diphyllobothrium mansoni (expérimental). Diphyllobothrium reptans. Cysticercus cellulosæ. Larve (Tetralhyridium ?). Clonorchis sinensis. Echinochasmus perfolatius.

Chat domestique. Trnia trnixformis. Dipylidium caninum. Diphyllobothrium replans. Clonorchis sinensis.

Zibethailurus nebulosus. Diphyllobothrium replans.

Viverra zibetha. Sparganum mansoni. 


\section{Oiseaux}

Poule domestique. Amobotania sphenoides. Raillielina (Raillietina) echinobolhrida. Raillietina (Raillietina) tetragona. Weinlandia cantaniana. Sparganum mansoni. Philophthalmus gralli. Prosthogonimus brauni.

Dindon domestique. Harmostomum (Postharmostomum) commutalum.

Numida meleargris. Sparganum mansoni.

Pavo muticus. Philophthalmus gralli.

Gennaeus nycthemerus. Sparganum mansoni.

Garzetta Garzetta. Ithyoclinostomum helerostomum.

Pigeon domestique d'Indochine. Sparganum mansoni.

Pigeon sp. de I'Inde. (Kasauli). Aporina delafondi. Raillietina (Raillietina) paucitesticulata. Raillielina (Raillietina) korkei. Colugnia cuneata var. nervosa. Weinlandia sp.

Carpophaga sylvatica. Raillielina (Raillietina) frayi.

Carpophaga œnea. Raillietina (Raillietina) carpophagi.

Canard domestique. Prosthogonimus rudolphii. Philophthalmus gralli.

Oie domestique. Philophthalmus gralli.

Dryonastes perspicillata. Sparganum mansoni.

Urocissa sinensis. Sparganum mansoni.

Upupa epops indica. Sparganum mansoni.

Corvus torquatus. Weinlandia serpentula. Prosthogonimus cuneatus.

\section{Reptiles}

Liolepis belliana. Oochoristica lagrangei.

Varanus sp. Scyphocephalus bisulcalus.

Mabuia siamensis. Lecithodendrium sp. Paradisloma mulabile.

Hypsirhina plumbea. Tetrathyridium sp.

Bungarus fasciatus. Proteocephalus naiz. Sparganum mansoni.

Zamenis mucosus. Sparganum mansoni. Teirathyridium sp. Slyphlodora lachesidis. Encyclometra caudata.

Naja tripudians. Sparganum mansoni.

Naja sp. Telralhyridium sp.

Python reticulatus. Bothridium pithonis. Bothridium pithonis var. parva.

\section{Batraciens}

Rana tigrina. Sparganum mansoni. Glyphtelmis lingualula. Prosolocus confusus.

\section{Poissons}

Anabas scandens. Melacercaria sp. (Ithyoclinostomum heterostomum ?). 


\section{BiBLIOGRAPHIE}

ANDo (A.). - Considerations relating to the History of the development of Sparganum mansoni, especially of its Dibothriocephalus decipiens. Far Eastern Assoc. Trop. Med. Trans. Sixth Biennial Congress, Tokyo, 1925, I, p. 421-422. Analysé in Trop. diseases Bull., XXIV, 1927, p. 179.

Ariola (V.). - Revisione della famiglia Bothriocephatides. str. Arch. de Parasitologie, III, 1900 , p. $369-484$, pl. VIII, IX, X.

BaCzynska (H.). - Etudes anatomiques et histologiques sur quelques nouvelles espèces de cestodes d'oiseaux. Thèse Sciences, Neuchâtel, 1914. Bull. Soc. neuchaléloise Sc. nat., XI, 1914, p. 187-239.

Baer (J.-G.). - Quelques cestodes d'oiseaux nouveaux et peu connus. Bull. Soc. neuchat. Sc. nat., XLIX, 1924, p. 138-154.

- Contributions to the Helminthic-fauna of south Africa. These Sciences, Neuchâtel, 11 th and 12 th. Report of the Director of Veterinary Education and Research. Pretoria, 1925, p. 61-136.

- Die Cestoden der Saügetiere Brasiliens. Abhand. Senkensbergs. Naturforsch. Gesell., 1927.

- Monographie de la famille Anoplocephalida. Bull. biol. France et Belgique, supplément X, 1927.

Baylis (H.-A.). - On two new species of the cestode genus Oochoristica from lizards. Parasitology, XI, 1919, p. 405-414, pl. XXI.

- Notes on some parasitic worms from east Africa. Annals Mag. Nat. Hist. (9), VI, 1920, p. 283-295.

BEDDARD (F.-E.). - Contributions to the anatomy and Systematic arrangement of the Cestoildea. On two new species belonging to the genera Oochoristica and Linstowia, with remarks upon those genera. Proc. zool. Soc_London (2), 1914, p. 263-283.

Bhalerao (G.-D.). - On the trematode parasites of a Watersnake. Tropidonotus piscator. Parasitology, XVIII, 1926, p. 4-13.

Brblet (A.). - Deux ans dans le Haut-Tonkin (région de Cao-Bang). Lille, 1896-1898.

BL เNchard (R.). - Notices helminthologiques (2 série). Mémoires Soc. zool. de France, IV, 1891, p. 420-485.

Вӧнм (L.-K.), - Beiträge zur Kenntnís tierischer Parasiten. C. B. f. Bakt. u. Paras. Orig., LXXXVII, 1922, p. 407-427, 1 pl.

Braun (M.). - Die Arten der Gattung Clinostomum Leidy. Zool. Jahr. Syst., XIV (1), 1900, 48 p., pl. I-II.

- Fascioliden der Vögel. Zool. Jahrb. Syst., XVI (1), 1902, 162 p., 8 pl.

Casaux. - Bull. Soc. méd. chirurg. d'Indochine, V, 1924, p. 374-377.

Casaux et Houdemgr (E.). - Note préliminaire sur les sparganoses humaines et animales au Tonkin. Bull. Soc, path. exot., XIX, 1926, p. 802-804.

Chander (A.-C.). - The helminthic Parasites of Cats in Calcutta and the relation

- of Cats to human helminthic Infections. Indian Journ. of med. Res., XIII, 1925, p. 213-227, pl. V-VI.

- New records on Bertiella satyri (Cestoda) in Man and apes. Parasilology, XVII, 1925, p. 421-425.

Cr.erc (W.). - Notes sur les cestodes d'oiseaux de l'Oural. C. B. f. Bakt. u. Parasit. Orig., XLII, 1906, p. 713-730.

Collın. - Annales d'hygiène et de méd. coloniales, $\mathrm{n}^{\circ} 1,1925$.

Diesing (C.-M.). - Systema helminthum. Vienne, 1850. 
Dollfus (R.-Ph.). - Observations sur la morphologie de Paradisloma mulabile (Molin) (Dicroçelide nouveau pour la faune française). Bull. Soc. zool. de France, XLVII, 1922, p. 387-404.

- Addition à la bibliographie de mes notes sur les Dicrocoliide et Paradistoma mutabile (Molin), Bull. Soc. zool. de France, XLVIII, 1923, p. 32.

- Sur un distome de Tropidonolus nalrix. Bull. Soc. zool. de France, XLIX, 1924 , p. $268-276$.

Evanno (Ch.-H.). - Contribution à l'élude de Sparganum mansoni, de Dibothriocephalus mansoni et de la pathogénie de la sparganose oculaire. Thèse méd. vetérin., Paris, $1927,39 \mathrm{p}$.

Fuhrmann (O.). - Die Anuplocepnaliden der Vögel. C. B. f. Bakt. u. Paras. Orig., XXXII, 1902, p. $122-147$.

- Neue Davaineiden. C. B. f. Bakt. n. Paras., XLIX, 1909, p. 94-124.

- Considérations générales sur les Davainea. Festschrift für Zschokke, no 27. Bâle, 1920.

- Two new species of reptilian Cestodes. Ann. trop. Med. and Parasitol., XVIII, 1924, p. 505-513.

Houdemer (E.). - Parasites des animaux domestiques ou sauvages du Tonkin. Bull. Soc. path. exot., XVIII, 1925, p. 343-350.

- Observations et recherches sur l hygiène et la pathologie animales du Tonkin. Thèse méd. vétér., Paris, 1927.

Isoвé (M.), - Présence de Trenia hydátigena Pallas, 1766 chez Macácus cynomolgus L. Annales de Parasitologie, II, 1924, p. 236-238.

Janicki (G.). - Uber zwei neue Arten des Genus Davainea. Arch. de Parasitologie, VI, 1902, p. 257-292.

Joyeux (Ch.). - Recherches sur la faune helminthologique africaine. Arch. Inst. Pasteur de Tunis, XII, 1923, p. 119-167 et p. 328-338.

- Cestodes des poules d'Indochine. Annales de Parasitologie, II, 1924, p. 314-318.

- Le Diphyllobothrium mansoni. Note préliminaire. Bull. Soc. palh. exot., $\mathrm{XX}, 1927$, p. 226-228.

Joyeux (Ch.) et BAER (J.-G.). - Recherches sur quelques espèces du genre Bothridium de Blainville, 1824. Annales de Parasitologie, V, 1927, p. 127-139.

Koвayashi (H.). - On the animal parasites in Korea. Japan med. World, V, 1925, p. $1-7$.

Kotran (A.). - Ueber Sparganum Raillieli Ratz und den zugehörigen geschlechtsreifen Bandwurm, Dibothriocephalus Raillieti Ratz. C. B. f. Bakt. $u$. Parasil. Orig., XC, 1923, p. 272-285.

Квавве (H.). - Voyage all Turkestan de A. P. Fedlchenko. Tome III, Cestodes, 1879 (en russe).

- Nye Bidrag til Kunskab om Fuglenes Bandelorme. 'In-4, Kjöbenhavn, 1882.

LeIPer (R.-T.). - Trans. Roy. Soc. trop. Med. and Hyg., XVII, 1923, p. 341.

Linstow (O. von). - Helminthes from the collection of the Colombo Museum Spolia zeylanica, III (11), 1906, p. 163-186, pl. I-III.

LüHE (M.). - Oochoristica nov. gen. Treniadarum (vorlaüfige Mittheilung). Zoolog. Anzeig., XXI, 1898, p. 650*653.

- Zur Auatomie und Systematik der Bothriocephaliden. Verhandl. der Deutsch. Zool. Ges., 1899, p. 30-35.

Mac Callum (G.-A.). - Studies in Helminthology. Zoopathologica, I (6), 1921, p. $140-284$.

Magalhaes (P.-S.). - Notes d'helminthologie brésilienne. Deux nouveaux ténias de la poule domestique. Archives de Parasitologie, I, 1898, p. 442-451.

Mathis (C.) et Leger (M.). - Recherches de parasilologie et de pathologie humaines et animales au Tonkin. In-8, 451 p., XIV pl. Paris, 1911. 
Mayhew (R.-L.). - Studies on the avian species of the cestode family Hymenolepidide. Illinois Biol. Mon., X (1), 1925, 125 p., IX pl.

MegGrtT (F.-J.). - On the life History of a reptilian tapeworm (Sparganum reptans). Ann. trop. Med. and Parasitol., XVIII, 1924, p. 195-204, pl. IX.

- The tapeworms of the Rangoon Pigeon. Parasitology, XVI, 1924, p. 303-312, pl. XIII.

The tapeworms of the domestic Fowl. Journ. of Burma Res. Soc., XV, 1926, p. 222-243, pl. III-V.

On the life-history of an amphibian tapeworm (Diphyllobotrium ranarum, Gastaldi). Ann. and Mag. nat., Hist.,.XVI, 1925, p. 654-655.

On a collection of burmese cestodes. Parasitology; XVHI, 1926, p. 230237 , pl. X.

Remarks on the Cestodes families Monticellide and Ichthyotaniida. Annals trop. Med. and Parasitol., XXI, 1927 a, p. 69-87.

On cestodes collected in Burma. Parasitology, XIX, 1927 b, p. 141-152.

Mogre (M.-A.). - Two new species of cestodes from indian Lizards. Rec. of the indian Mus., XXVIII, 1926, p. 53-60.

Motais (F.). - La sparganose oculaire en Annam. Bull. Soc. path. exot., XIII, 1920 , p. 215-222,

Muto (M.) et Mrвaнa (Y). - On the origin of a species of Cestodes - the adult form of Sparganum mansoni - that prevails among the cats in a village of Okayama Prefecture. Tokyo Iji Shinshi, n० 2217, mars 1921. Analysé in Trop. dis. Bull., XX, 1923, p. 224.

Nybelin (O.). - Anatomische-systematische Studien über Pseudophyllideen. Göleborgs kungl. Vetenkaps-och Vitterhels-Samhälles Handlingar födjen, XXVI (1), 1922.

Okumura (T.). - An experimental study on the life-history of Sparganum mansoni. Kilasato Arch. of experim. Med., III, 1919, p. 190-196, I pl.

Railliet (A.). - Les cestodes des oiseaux domestiques. Recueil de Médecine vétérinaire, XCVII, 1921, p. 185-205.

- Les helminthes des animaux domestiques et de l'homme en Indochine. Bull. Soc. zoolog. de France, $1^{\text {re }}$ partie, XLIX, 1924, p. 588-608. 2e partie, L, 1925, p. $7-26$.

Ralltete (A.), Hexny (A.) et Bauche (J.). - Sur les amphistomiens des ruminants domestiques de l'Annam. Recueil de Méd. vétér., XCI, 1914, p. 195-201.

Railliet (A.) et Lucet (A.). - Sur lidentité du Davainea oligophora Magalhães, 1898 et du Trenia cantaniana Polonio, 1860. Archives de Parasitologie, II, 1899, p. 144-146.

Ransom (B.-H.). - The tanioid cestodes of north american Birds. Smilh. Inst. U. S. nat. Mus. Bull., 69,1909 , p. $36-41$.

Rizzo (A.). - La fauna elmintologica dei rettili nella provincia di Catania. Arch. de Parasit., VI, 1902, p. 26-41.

Rudin (E.). - Oochoristica truncala Krabbe. Zool. Anzeiger, XLYII, 1916, p. 75-78 et 81-85.

Skriabine (K.-I.). - Vogeltrematoden aus Russich Turkestan. Zool. Jahrb. Syst., XXXIII (3), 1913, p. 351-388, pl. XIII-XIV.

- Trématodes des oiseaux domestiques (én russe). In-80 de 64 p. Moscou, 1923.

Sonsino (P.). - Studi e notizie elmintologiche. Proc. verb. Soc. Toscana di Sc. Natur., 12 maggio 1889 (a), p. 224-237.

- Studi e notizie elmintologiche. Ibid. 17 luglio 1889 (b), p. 273-285.

- Notizie di parassiti. Ibid, 18 gennaio 1891.

Southwel. (T.). - Cestodes of Indian Poultry. Annals trop. Med. and Parasilol., XV, 1921, p. 161-166. 
Southwell (T.). - Cestodes from african Rats. Ann. trop. Med. and Parasit., XV, 1921 , p. $167-168$.

- Cestodes in the collection of the indian Museum. Annals trop. Med. and Parasitol., XVI, 1922, p. 127-152.

A monograph of the Tetraphyllidea with notes on relate cestodes. Memoir no 2. Liverpool School of trop. Med. University Press Liverpool, 1925, 368 p.

Stiles (C.-W.) et Orleman (M.). - La nomenclature des genres de cestodes Raillietina, Rãnsomia et Johnstonia. Annales de Parasitologie, IV, 1926, p. $65-67$.

Travassos (L.). - Contribuiçôes para o conhecimento dos helminthos dos batraquios do Brasil. Sciencia Medica, II (11), 1924, p. 1-12.

Wagener (G.-R.). - Enthelminthien, no III. Arch. f. Anatomie, Physiologie u. wiss. Med., 1852, p. 555-569, pl. XVI.

Weithofer (M.). - Vogelcestoden aus Senaar und Kordofan. Wissens. Erg. Werner's zool. Exp. anglo-ägypt. Sudan (Kordofan), 1914. Kais. Akad. Wiss. Wien. Math. Natur. wiss. Klas, , 1er décembre 1916.

Witenberg. - Versuch einer Monographie der Trematodenunterfamilie Harmostomina. Zool. Jahrb. Syst., LI, 1925, p. 169-254, pl. I-II.

Woodland (W.-N.-F.). - On Proteocephalus marenzelli, $P$. naia, and $P$. viperis. Annals trop. Med. and Parasitol., XIX, 1925, p. 265-279.

Yamata(S.). - Contribution to the life history of Bothriocephalus lalus. Taiwan Igakukai Zassi, no 168, 28 octobre 1916. Résumé in : China med. Journ:, XXXI, 1917, p. 179.

Yoshid (S.). = On three new species of Hymenelopis found in Japan. Annotationes zoologica japonenses, VII, 1910, p. 314-318.

- The occurence of Bothriocephalus liguloides Leuckart, with especial reference to its development. Journ. of Parasitol., III, 1917, p. 170-176.

- On the morphology of the adult worm of Sparganum mansoni found in the Frog and other Animals. Tokyo Iji-Shinshi, n० 2271-2272, mars-avril 1922. Analysé in Trop. dis. Bull., 1923, p. 223.

Zschокке (F.). - Zeits. f. wiss. Zool., LXXXIII, 1905, p. 60.

Laboratoire de Parasilologie de la Faculté de médecine de Paris. 